\title{
REGULARITY, NORMALITY AND MULTIFUNCTIONS
}

\author{
JAMES E. JOSEPH
}

ABSTRACT. In this note we give several characterizations of regularity and normality in terms of weakly upper-semicontinuous multifunctions.

1. Introduction. Characterizations of regularity and normality of topological spaces in terms of multifunctions have been given by Engelking [E], Franklin and Sorgenfrey [FS] and Wyler [W]. In this article we use multifunctions to obtain several new characterizations of regularity and normality. The definitions of regularity and normality are not assumed to include $T_{1}$.

2. Some preliminaries. We will denote the closure of a subset $A$ of a space by $\mathrm{cl}(A)$ and the family of open sets which contain $A$ by $\Sigma(A)$. If $X$ is a set we will represent the diagonal of $X \times X$, i.e. $\{(x, x): x \in X\}$, by $\Delta$. If $X$ is a set, $x_{0} \in X$, and $\Omega$ is a filterbase on $X$, then $X$ with the topology $\{V \subset X$ : $x_{0} \notin V$ or $F \subset V$ for some $\left.F \in \Omega\right\}$ will be denoted by $X\left(x_{0}, \Omega\right)$. A multifunction from a set $X$ to a set $Y$ is a function from $X$ to $2^{Y}-\{\varnothing\}$, where $2^{Y}$ is the power set of $Y$. We will write $\Phi: X \rightarrow Y$ to denote that $\Phi$ is a multifunction from $X$ to $Y$. A multifunction $\Phi: X \rightarrow Y$ on a space $X$ into a space $Y$ is upper-semicontinuous (u.s.c.) at $x \in X$ if for each $W \in \Sigma(\Phi(x))$ in $Y$ there is a $V \in \Sigma(x)$ in $X$ with $\Phi(V) \subset W$; $\Phi$ is upper-semicontinuous (u.s.c.) if $\Phi$ is u.s.c. at each $x \in X$. Levine [L] has called a function $\Phi: X \rightarrow Y$ from a space $X$ into a space $Y$ weakly-continuous at $x \in X$ if for each $W \in \Sigma(\Phi(x))$ in $Y$ there is a $V \in \Sigma(x)$ in $X$ with $\Phi(V) \subset \operatorname{cl}(W)$; the function $\phi$ is weaklycontinuous if $\Phi$ is weakly-continuous at. each $x \in X$. Smithson [S] has extended Levine's definition to multifunctions, defining a multifunction $\Phi$ : $X \rightarrow Y$ from a space $X$ to a space $Y$ to be weakly upper-semicontinuous (w.u.s.c.) at $x \in X$ if for each $W \in \Sigma(\Phi(x))$ in $Y$ there is a $V \in \Sigma(x)$ in $X$ with $\Phi(V) \subset \mathrm{cl}(W) ; \Phi$ is weakly upper-semicontinuous (w.u.s.c.) if $\Phi$ is w.u.s.c. at each $x \in X$. It is not difficult to establish that a continuous (u.s.c.) function (multifunction) is weakly-continuous (w.u.s.c.). It is known that a weakly-continuous function need not be continuous. However, a weaklycontinuous function into a regular space is continuous. The following example shows that a w.u.s.c. multifunction into a regular space need not be u.s.c.

Received by the editors May 31, 1977 and, in revised form, October 6, 1977.

AMS (MOS) subject classifications (1970). Primary 54D10, 54D15; Secondary 54C60, 54C05.

Key words and phrases. Multifunctions, regularity, normality, upper-semicontinuous, weakly upper-semicontinuous.

(1) American Mathematical Society 1978 
2.1. Example. Let $Y$ consist of those points $(x, y)$ of the Euclidean plane for which $y \geqslant 0$. Let $Y_{0}=Y-\{(x, 0):(x, 0) \in Y\}$. For each $z \in Y_{0}$ the base for the open sets is the interiors of circles with center at $z$. For $(x, 0)$ we choose as a base sets consisting of the point $(x, 0)$ and the interior of a circle tangent to the $X$-axis at $(x, 0)$. It is well known that $Y$ with this topology is regular [P, p. 88]. Let $\Omega$ be the filterbase of finite complements on $Y$, let $z_{0} \in Y$, and define $\Phi: Y\left(z_{0}, \Omega\right) \rightarrow Y$ by $\Phi\left(z_{0}\right)=Y_{0}$ and $\Phi(z)=z$ if $z \neq z_{0}$. The multifunction $\Phi$ is clearly u.s.c. at each $z \neq z_{0}$ and is w.u.s.c. at $z_{0}$ since if $W \in \Sigma\left(\Phi\left(z_{0}\right)\right)$ we have cl $(W)=Y$. On the other hand, $Y_{0} \in \Sigma\left(\Phi\left(z_{0}\right)\right)$ and for each $V \in \Sigma\left(z_{0}\right)$ in $Y\left(z_{0}, \Omega\right)$ some $(x, 0) \in V-\left(Y_{0} \cup\left\{z_{0}\right\}\right)$. Thus, $\Phi$ is not u.s.c. at $z_{0}$.

3. Characterizations of regularity. Let $X$ and $Y$ be spaces, $\Phi: X \rightarrow Y$ be a function and $\alpha: X \rightarrow Y$ be a multifunction. Using the definition of Weston [W] as a model we define the cluster set of $\alpha$ at $x \in X$ to be $C(\alpha ; x)=$ $\bigcap_{\Sigma(x)} \mathrm{cl}(\alpha(V))$. We use the notations $R(\Phi, \alpha, X, Y)=\{x \in X: \Phi(x) \in$ $\operatorname{cl}(\alpha(x))\}$ and $R(\Phi, \alpha, X \times X, Y)=\{(a, b) \in X \times X: \Phi(a) \in \operatorname{cl}(\alpha(b))\}$.

3.1. THEOREM. If $\alpha: X \rightarrow Y$ is a w.u.s.c. multifunction and $Y$ is regular then $C(\alpha ; x)=\operatorname{cl}(\alpha(x))$ for each $x \in X$.

Proof. It is obvious that $\mathrm{cl}(\alpha(x)) \subset C(\alpha ; x)$ for each $x \in X$. On the other hand, if $x \in X$ and $W \in \Sigma(\alpha(x))$ in $Y$ there is a $V_{W} \in \Sigma(x)$ in $X$ with $\alpha\left(V_{W}\right) \subset \mathrm{cl}(W)$. So,

$$
C(\alpha ; x) \subset \bigcap_{\Sigma(\alpha(x))} \mathrm{cl}\left(\alpha\left(V_{W}\right)\right) \subset \bigcap_{\Sigma(\alpha(x))} \mathrm{cl}(W) .
$$

Since $Y$ is regular we have $\cap_{\Sigma(\alpha(x))} \operatorname{cl}(W)=\operatorname{cl}(\alpha(x))$. The proof is complete.

3.2. Theorem. $Y$ is regular if $C(\alpha ; x)=\operatorname{cl}(\alpha(x))$ for each space $X$, u.s.c. multifunction $\alpha: X \rightarrow Y$, and $x \in X$.

Proof. If $Y$ is not regular there is a nonempty closed subset $F$ of $Y$ such that $\cap_{\Sigma(F)} \operatorname{cl}(W) \neq F$. Choose $x_{0} \in F$ and let $\Omega=\Sigma(F)$. Define $\alpha: Y\left(x_{0}, \Omega\right) \rightarrow Y$ by $\alpha\left(x_{0}\right)=F$ and $\alpha(x)=x$ otherwise. It is obvious that $\alpha$ is u.s.c. at each $x \neq x_{0}$. If $W$ $\in \Sigma\left(\alpha\left(x_{0}\right)\right)$ in $Y$ then $W \cup\left\{x_{0}\right\} \in \Sigma\left(x_{0}\right)$ in $Y\left(x_{0}, \Omega\right)$ and $\alpha\left(W \cup\left\{x_{0}\right\}\right)=W$; so $\alpha$ is u.s.c. at $x_{0}$. However $C\left(\alpha ; x_{0}\right) \neq \operatorname{cl}\left(\alpha\left(x_{0}\right)\right)$. This means that $Y$ does not satisfy the hypothesis of the theorem and the proof is complete.

Combining Theorems 3.1 and 3.2 we obtain the following characterization of regularity.

3.3. Corollary. A space $Y$ is regular if and only if for each space $X$ and w.u.s.c. multifunction $\alpha: X \rightarrow Y, C(\alpha ; x)=\operatorname{cl}(\alpha(x))$ for each $x \in X$.

Theorem 3.4 gives other characterizations of regularity.

3.4. THEOREM. The following statements are equivalent for a space $Y$. 
(a) $Y$ is regular.

(b) $R(\Phi, \alpha, X \times X, Y)$ is closed in $X \times X$ for each space $X$, continuous function $\Phi: X \rightarrow Y$ and w.u.s.c. multifunction $\alpha: X \rightarrow Y$.

(c) $R(\Phi, \alpha, X, Y)$ is closed in $X$ for each space $X$, continuous function $\Phi$ : $X \rightarrow Y$ and w.u.s.c. multifunction $\alpha: X \rightarrow Y$.

Proof that (a) imples (b). Let $(a, b) \in(X \times X)-R(\Phi, \alpha, X \times X, Y)$. Then $\Phi(a) \notin \mathrm{cl}(\alpha(b))$ and, since $Y$ is regular, there are sets $V \in \Sigma(\Phi(a))$, $W \in \Sigma(\operatorname{cl}(\alpha(b)))$ in $Y$ with $\operatorname{cl}(V) \cap \operatorname{cl}(W)=\varnothing$. Since $\Phi$ is continuous and $\alpha$ is w.u.s.c. there are sets $A \in \Sigma(a), B \in \Sigma(b)$ in $X$ with $\Phi(A) \subset V$ and $\alpha(B) \subset \operatorname{cl}(W)$. Then $A \times B \in \Sigma((a, b))$ in $X \times X$ and $(A \times B) \cap$ $R(\Phi, \alpha, X \times X, Y)=\varnothing$. This completes the proof that (a) implies (b).

Proof that (b) Implies (c). The restriction of the projection, $\pi_{x}: X \times X \rightarrow$ $X$, to $\Delta$ is a homeomorphism, $R(\Phi, \alpha, X \times X, Y) \cap \Delta$ is closed in $\Delta$ and $R(\Phi, \alpha, X, Y)=\pi_{x}(R(\Phi, \alpha, X \times X, Y) \cap \Delta)$. The proof that (b) implies (c) is complete.

Proof that (c) IMPlies (a). Assume that $Y$ is not regular; then there is a nonempty closed subset $F$ in $Y$ and an $x_{0} \in Y-F$ such that $\Omega=\{V \cap W$ : $\left.V \in \Sigma(F), W \in \Sigma\left(x_{0}\right)\right\}$ is a filterbase on $Y$. Choose $y_{0} \in F$. Define $\Phi, \alpha$ : $Y\left(y_{0}, \Omega\right) \rightarrow Y$ as follows: $\Phi(x)=x$ if $x \neq y_{0}$ and $\Phi\left(y_{0}\right)=x_{0} ; \alpha(x)=x$ if $x \neq y_{0}$ and $\alpha\left(y_{0}\right)=F$. Clearly, $\Phi$ and $\alpha$ are both continuous at each $x \neq y_{0}$. We see also that $\alpha$ is u.s.c. ( $\Phi$ is continuous) at $y_{0}$ since if $W \in \Sigma\left(\alpha\left(y_{0}\right)\right)$ $\left(\Sigma\left(\Phi\left(y_{0}\right)\right)\right)$ in $Y$, then $W \cup\left\{y_{0}\right\} \in \Sigma\left(y_{0}\right)$ in $Y\left(y_{0}, \Omega\right)$; and $\alpha\left(W \cup\left\{y_{0}\right\}\right) \subset$ $W\left(\Phi\left(W \cup\left\{y_{0}\right\}\right) \subset W\right)$. We observe that $R(\Phi, \alpha, X, Y)=Y-\left\{y_{0}\right\}$. If $V$ $\in \Sigma\left(x_{0}\right)$, and $W \in \Sigma(F)$ in $Y$, then $(V-F) \cap W \neq \varnothing$, and for $x \in(V-$ $F) \cap W$ we have $\Phi(x)=\alpha(x)=x$. So $y_{0} \in \operatorname{cl}(R(\Phi, \alpha, X, Y))$ in $Y\left(y_{0}, \Omega\right)$. The proof that (c) implies (a) is complete.

The proof of the theorem is complete.

4. Characterizations of normality. If $X, Y$ are spaces, and $\Phi, \alpha: X \rightarrow Y$ are multifunctions, we use the notations

$$
\begin{gathered}
N(\Phi, \alpha, X \times X, Y)=\{(a, b) \in X \times X: \operatorname{cl}(\Phi(a)) \cap \operatorname{cl}(\alpha(b)) \neq \varnothing\}, \\
N(\Phi, \alpha, X, Y)=\{x \in X: \operatorname{cl}(\Phi(x)) \cap \operatorname{cl}(\alpha(x)) \neq \varnothing\} .
\end{gathered}
$$

4.1. THEOREM. The following statements are equivalent for a space $Y$.

(a) $Y$ is normal.

(b) $N(\Phi, \alpha, X \times X, Y)$ is closed in $X \times X$ for each space $X$ and all w.u.s.c. multifunctions $\Phi, \alpha: X \rightarrow Y$.

(c) $N(\Phi, \alpha, X, Y)$ is closed in $X$ for each space $X$ and all w.u.s.c. multifunctions $\Phi, \alpha: X \rightarrow Y$.

Proof that (a) implies (b). Let $(a, b) \in(X \times X)-N(\Phi, \alpha, X \times X, Y)$. Then $\operatorname{cl}(\Phi(a)) \cap \operatorname{cl}(\alpha(b))=\varnothing$. Since $Y$ is normal there are sets $V \in$ $\Sigma(\operatorname{cl}(\Phi(a))), W \in \Sigma(\operatorname{cl}(\Phi(b)))$, in $Y$ satisfying $\operatorname{cl}(V) \cap \operatorname{cl}(W)=\varnothing$. Since $\Phi$ and $\alpha$ are w.u.s.c. there are sets $A \in \Sigma(a), B \in \Sigma(b)$, in $X$ with $\Phi(A) \subset \operatorname{cl}(V)$ and $\Phi(B) \subset \operatorname{cl}(W)$. Then $A \times B \in \Sigma((a, b))$ in $X \times X$ and $(A \times B) \cap$ 
$N(\Phi, \alpha, X \times X, Y)=\varnothing$. This completes the proof that (a) implies (b).

Proof that (b) IMPLies (c). The proof is similar to the proof of (b) implies (c) of Theorem 3.4.

Proof that (c) IMPLies (a). Assume that $Y$ is not normal; then there are nonempty closed sets $F, H \subset Y$ with $F \cap H=\varnothing$ such that $\Omega=\{V \cap W$ : $V \in \Sigma(F), W \in \Sigma(H)\}$ is a filterbase on $Y$. Choose $x_{0} \in F$ and define $\Phi, \alpha$ : $Y\left(x_{0}, \Omega\right) \rightarrow Y$ by $\Phi\left(x_{0}\right)=H, \alpha\left(x_{0}\right)=F$ and $\Phi(x)=\alpha(x)=x$ otherwise. We see by arguments similar to those above that $\Phi$ and $\alpha$ are u.s.c. Since $\operatorname{cl}\left(\Phi\left(x_{0}\right)\right)=H$ and $\operatorname{cl}\left(\alpha\left(x_{0}\right)\right)=F$ we see that $x_{0} \notin N\left(\Phi, \alpha, Y\left(x_{0}, \Omega\right), Y\right)$. Let $A \in \Sigma\left(x_{0}\right)$ in $Y\left(x_{0}, \Omega\right)$. There are sets $V \in \Sigma(F), W \in \Sigma(H)$ in $Y$ satisfying $\varnothing \neq(V-H) \cap(W-F) \subset A$. If $x \in(V-H) \cap(W-F)$ we have $\Phi(x)=\alpha(x)=x$; so $x \in N\left(\Phi, \alpha, Y\left(x_{0}, \Omega\right), Y\right)$ and, consequently, $x_{0}$ $\in \operatorname{cl}\left(N\left(\Phi, \alpha, Y\left(x_{0}, \Omega\right), Y\right)\right)$ in $Y\left(x_{0}, \Omega\right)$. This is a contradiction of statement (c) so the proof that (c) implies (a) is complete.

The proof of the theorem is complete.

Acknowledgement. The author thanks Professor M. Solveig Espelie for extremely helpful discussions in the preparation of this paper.

\section{REFERENCES}

[E] R. Engelking, Quelques remarques concernant les opérations sur les fonctions semicontinues dans les espaces topologiques, Bull. Acad. Polon. Sci. Ser. Sci. Math. Astronom. Phys. 11 (1963), 719-726.

[FS] S. P. Franklin and R. H. Sorgenfrey, Closed and image closed relations, Pacific J. Math. 19 (1966), 433-439.

[L] Norman Levine, A decomposition of continuity in topological spaces, Amer. Math. Monthly 68 (1961), 44-46. MR 23 \# A3548.

[P] W. J. Pervin, Foundations of general topology, Academic Press, New York, 1964.

[S] R. E. Smithson, Almost and weak continuity for multifunctions (preprint).

[W] O. Wyler, A characterization of regularity in topology, Proc. Amer. Math. Soc. 29 (1971), 588-590.

Department of Mathematics, Howard University, Washington, D.C. 20059 